2017-03-07

\title{
Class Type Separation of the Polar and Apolar Components of Petroleum
}

\author{
Robson, WJ
}

http://hdl.handle.net/10026.1/8599

10.1021/acs.analchem.6b04202

ANALYTICAL CHEMISTRY

All content in PEARL is protected by copyright law. Author manuscripts are made available in accordance with publisher policies. Please cite only the published version using the details provided on the item record or document. In the absence of an open licence (e.g. Creative Commons), permissions for further reuse of content should be sought from the publisher or author. 
Please note: This is a pre-publication copy of the final article published in Analytical Chemistry. Readers are advised to consult the final published article before citing the work.

\title{
Class Type Separation of the Polar and Apolar Components of Petroleum
}

\author{
William J. Robson ${ }^{\dagger}$, Paul Sutton ${ }^{\dagger}$, Paul McCormack ${ }^{\dagger}$, Neil P. Chilcott ${ }^{\dagger \dagger}$, and Steven J. Rowland ${ }^{\dagger *}$ \\ ${ }^{\dagger}$ Petroleum and Environmental Geochemistry Group, Biogeochemistry Research Centre, University of Plymouth, UK, \\ fax: +44(0)1752 584710, e-mail: srowland@plym.ac.uk
}

*Kernow Analytical Technology Limited, Cornwall, UK.

\begin{abstract}
Identification of the heteroatom (nitrogen, sulphur, and oxygen)- containing compounds of petroleum is of key importance when considering industrial and environmental issues associated with crude oil production. The more commonly performed methods of crude oil fractionation are often insufficient in the extent to which they separate oils, not allowing defined 'molecular' fractions to be obtained. Methods capable of performing a class type separation are uncommon and are often extensive, and resource and time intensive. Here we report a method for the separation of crude oils into discrete compound classes. The method utilizes both ion exchange and normal phase chromatography to generate fractions of saturate hydrocarbons, aromatic hydrocarbons, basic compounds, naphthenic acids and other oxygen containing species, carbazoles, sulfones, and thiophenes from small crude oil samples $(\sim 0.5 \mathrm{~g})$. Method validation with a suite of model compounds has shown the fractions to be well-defined; with classes of model compounds isolated within discrete fractions. Application of the method to five crude oils of varying API gravity $\left(12.1-38.3^{\circ}\right)$ demonstrates a potential for wide-ranging use. Sample recoveries were high (93-103\%) once corrected for evaporative losses, as was repeatability, demonstrated by triplicate analyses of model compound mixtures, oils spiked with model compounds and oils alone. Separation selectivity was further demonstrated by application of the scheme to the Alaska North Slope (ANS) crude oil and analysis of fractions by comprehensive two dimensional gas-chromatography mass-spectrometry (GC $\times$ GC-MS) and/or liquid-chromatography high-resolution accurate-mass mass-spectrometry methods (LC-HRAM-MS). Isolation of discrete fractions then allowed excellent separation (by LC and GC methods) of carbazole, dibenzothiophene, fluorenones, xanthones, and quinoline fractions. Individual parent and $\mathrm{C}_{1-5}$ alkyl homologs were easily separated (GC $\times \mathrm{GC}-\mathrm{MS}$ ) allowing high quality mass spectra (EI) to be obtained for the individual compounds in many cases. Analysis of fractions by GC $\times$ GC-MS also allowed a series of thioxanones to be identified.
\end{abstract}

\section{INTRODUCTION}

The inherent molecular complexity of crude petroleum is such that detailed compositional investigations must ideally be preceded by some form of pre-fractionation. Such investigations into petroleum composition are often focused on the nitrogen, sulphur, and oxygen (NSO) containing or so-called 'polar' compounds, with interest in these heterofunctionalised compounds covering many subject areas. For instance, nitrogen containing compounds in crude oils are present as both neutral (indoles, carbazoles, and pyrroles), and basic nitrogen (pyridinic and quinolines) species. Interests in these compounds are primarily focused on their tendency to poison processing catalysts ${ }^{[1-3]}$, reduce product stability ${ }^{[4]}$, and on their associated toxicity in environmental scenarios (i.e. ${ }^{[5]}$ ). Likewise, major sulphur containing compounds in crude oils are sulfidic and thiophenic compounds; both are also of environmental concern ${ }^{[6]}$, and the latter are thought to be molecular tracers for migration distances ${ }^{[7]}$. Oxygen-containing compounds are predominantly present as both naphthenic acids and phenols ${ }^{[8]}$, but also as ketones; including functionalised aromatic molecules ${ }^{[9,10]}$. Naphthenic acids (NA) are responsible for a number of issues throughout oil extraction and refining processes. These include: corrosion during refining, storage and transportation ${ }^{[11,12]}$, toxicity ${ }^{[13,14]}$, and the formation of calcium naphthenate deposits in production equipment and refineries ${ }^{[15]}$. To facilitate investigations into such subject areas, crude oils are routinely fractionated into bulk classes used to represent properties of the oil (e.g. asphaltenes, defined as a solubility class, total acid number (TAN) and total base number (TBN) by titration). The last several decades have seen the development of a substantial number of methods for crude oil separation. The most common are the so-called SARA (Saturates, Aromatics, Resins, and Asphaltenes) type separations. After the initial SARA separation was introduced by Jewell et.al. ${ }^{[16]}$, significant numbers of SARA separations were developed (e.g. references ${ }^{[17-28]}$ ), utilising a variety of solid phases, techniques, and detectors. The drawbacks of these methodologies are well known (summarised ${ }^{[29,30]}$ ), with commonly identified issues including method inconsistencies, poor precision and repeatability, cross-contamination, and obligatory de-asphalting steps. Whilst recent developments in SARA methodologies do allow separation of whole crudes (utilising PTFE packed columns and heptane solutions to precipitate asphaltenes online) ${ }^{[31-33]}$, in practice few labs are equipped to perform such separations. Although employing SARA methods can quickly provide fractions of saturated hydrocarbons for rapid gas chromatography (GC) analysis, compositional studies into the more complex resin and asphaltene fractions require further fractionations and studies commonly employ ultra-high resolution mass spectrometers (uHRMS; e.g. resolving power $>100,000)^{[34-36]}$ to attempt to overcome the complexity. The use of uHRMS (e.g. FT-ICR-MS and orbitrap MS) is regularly applied in petroleum analysis but does not provide information concerning molecular structures and even assigning functional groups can be difficult. For example, in excess of 20,000 molecular formulas can be assigned in a single mass spectrum (e.g. 
Please note: This is a pre-publication copy of the final article published in Analytical Chemistry. Readers are advised to consult the final published article before citing the work.

${ }^{[37,38]}$ ) but no information on isomeric structures for the assigned formula are then available and functionality must be predicted. As issues such as toxicity ${ }^{[39]}$, catalyst poisoning ${ }^{[40]}$, and deposition ${ }^{[41]}$ are often structurally dependent, the importance of a complementary combined approach, enabling chemical, molecular and structural characterisation must be stressed.

Less commonly reproduced are the extensive, class-type separations ${ }^{[42,43]}$, and more targeted crude oil separations (e.g. ${ }^{[10,44-}$ $\left.{ }^{46]}\right)$. Historically, class-type separations are laborious, generating large numbers of fractions (e.g. ${ }^{[43]}: 36$ fractions). Many normal phase separations result in significant losses due to irreversible adsorption (summarised ${ }^{[47]}$ ) and require asphaltene precipitation prior to separation. Despite the drawbacks, this approach has many benefits, the most significant being the ability to collect fractions based on compound class. Many of these methods utilise both ion exchange (IEX) and normal phase chromatography to generate fractions. Those methods utilising IEX more commonly apply anion exchange (AX) chromatography ${ }^{[43,48-51]}$ as the initial point of separation and more rarely, cation exchange (CX) chromatography ${ }^{[15,44]}$. Removal/isolation of higher polarity/ionic species results in substantially reduced losses due to irreversible adsorption to normal phases and also removes the need for de-asphalting steps, thus allowing whole crudes to be separated. Despite the common implementation of a primary AX step, the effectiveness of the CX step at reducing the matrix complexity of samples prior to separation by AX, whilst maintaining integrity of acid fractions has been demonstrated ${ }^{[15]}$. The consequence of such a large number of separations is the accumulation of substantial data sets for different crude oils that are to a large extent, incomparable. The limited specificity and poor recovery provided by SARA separations and the extensive number of fractions arising from the more compound specific separations suggests that a cheap and reproducible method for the separation of compound classes from crude oils would be advantageous.

Here we report a new method for the comprehensive separation of crude oils, which separates oils into fractions based on the functionality of the various compound classes in oils. We employed the use of authentic compounds representative of many compound classes known to be present in crude oils. This allowed the creation of an optimised scheme in which class location could be reliably predicted for crude oil separations. The developed separation allows collection of 12 separate fractions from crude oils samples: saturates, aromatics, basic compounds, NA and other oxygen containing species, carbazoles, sulfones, and thiophenes.

\section{EXPERIMENTAL DETAIL}

Extraction solvents, authentic compounds and samples- Solvents used for separations were at least HPLC grade (Rathburn Chemicals, Scotland): $n$-hexane, dichloromethane (DCM), tetrahydrofuran (THF), and toluene, except diethyl ether (DEE; reagent grade, Rathburn Chemicals, Scotland). Water $\left(\mathrm{H}_{2} \mathrm{O}\right)$ was $18.2 \mathrm{M} \Omega \mathrm{cm}^{-1}$ grade (Elga Maxima Analytical; Elga Ltd., U.K.). Acid-base modifiers were reagent grade, formic acid (FA, 95\%; Sigma-Aldrich Company Ltd., U.K.) and ammonium hydroxide (35\%; Fisher Scientific, U.K.). All model compounds were purchased from suppliers at $\geq 97 \%$ purity (confirmed by GC-MS analysis): adamantane carboxylic acid, $5 \beta$ cholanic acid, dibenzyl disulfide, dibenzyl sulfoxide, dibenzofuran, dibenzofuran-4-carboxylic acid, dibenzothiophene sul-

fone, 4-dibenzothiophene carboxylic acid, fluorenone, 5-nitroindole, 4-pentylbicyclo[2.2.2] octane-1-carboxylic acid, 2phenyl phenol, phenanthrene and xanthone (Sigma-Aldrich Company Ltd., Poole, U.K.), benzo[ $h]$ quinoline, dibenzyl sulfoxide, dibenzothiophene, fluoranthene (Acros Organics, Belgium), and benzo[a]pyrene (Supelco Inc., Bellefonte, USA). Crude oils were obtained as follows: Alaska North Slope (ANS), Kuwait Blend and Brent crude from British Petroleum. Bonga crude was provided by the Nigerian National Petroleum Company and Tijuana Pesado crude from Royal Dutch Shell (Table S2 lists bulk crude oil properties).

Method of Crude Oil Separation- Crude oil fractionations were based on a modification of the method used by Sutton and Rowland ${ }^{[15]}$ and the sequential procedure is shown in Figure 1. Crude oil was first homogenised (1 hr, Stuart SSL1 Orbital Shaker, $100 \mathrm{rpm}$ ) and $\sim 0.5 \mathrm{~g}$ was accurately weighed into a 7 $\mathrm{mL}$ glass vial. The crude oil was diluted in $\mathrm{DEE} / \mathrm{H}_{2} \mathrm{O} / \mathrm{FA}$ $(7 \mathrm{~mL} ; 0.1 \% / 0.1 \% ; \mathrm{v} / \mathrm{v} / \mathrm{v})$ and mixed (vortex, 10s) before being introduced onto a sequentially pre-conditioned $(20 \mathrm{~mL}, 0.1 \%$ FA (aq); $10 \mathrm{~mL}, \mathrm{H}_{2} \mathrm{O} ; 20 \mathrm{~mL}$, THF; $20 \mathrm{~mL}$, toluene; $20 \mathrm{~mL}$, DEE; flush dried between eluents) strong cation exchange (SCX), solid phase extraction (SPE) cartridge (DSC-SCX, 12 mL, 2g; Sigma-Aldrich Company Ltd., Poole, U.K.). Fractions were sequentially eluted from the SCX cartridge under vacuum $\left(-0.1\right.$ bar) with the following solvents: DEE/ $\mathrm{H}_{2} \mathrm{O} / \mathrm{FA}$ (20mL;0.1\%/0.1\%; v/v/v; SCX0), toluene (20 mL; SCX1), THF (20mL; SCX2), THF/ $\mathrm{H}_{2} \mathrm{O} / \mathrm{ammonia}$ (20mL;0.1\%/2\%; $\mathrm{v} / \mathrm{v} / \mathrm{v} ; \mathrm{SCX} 3)$, and $\mathrm{THF} / \mathrm{H}_{2} \mathrm{O} / \mathrm{ammonia}(20 \mathrm{~mL} ; 0.1 \% / 5 \%$; v/v/v; SCX4), flush dried between eluents. Solvents were subsequently removed under nitrogen at suitable temperatures (DEE, THF: $30{ }^{\circ} \mathrm{C}$, toluene: $\left.70^{\circ} \mathrm{C}\right)$. After drying, fraction SCX0 was diluted with DEE/ $\mathrm{H}_{2} \mathrm{O} / \mathrm{ammonia}(7 \mathrm{~mL} ; 0.1 \% / 0.1 \%$; v/v/v) and applied to a pre-conditioned $(20 \mathrm{~mL}, 1 \%$ ammonia (aq); $10 \mathrm{~mL}$ $\mathrm{H}_{2} \mathrm{O} ; 20 \mathrm{~mL}$ THF; $20 \mathrm{~mL}$ toluene; $20 \mathrm{~mL}$ DEE) strong anion exchange (SAX) SPE cartridge (DSC-SAX, 12 mL, 2g, SigmaAldrich Company Ltd., Poole, U.K.). Fractions were sequentially eluted from the SAX cartridge under vacuum (-0.1 bar) in the following solvents: DEE/ $\mathrm{H}_{2} \mathrm{O} / \mathrm{ammonia} \quad(20$ $\mathrm{mL} ; 0.1 \% / 0.1 \%$; v/v/v; SAX0), toluene (20 mL; SAX1), THF $(20 \mathrm{~mL} ; \mathrm{SAX} 2)$, and $\mathrm{THF} / \mathrm{H}_{2} \mathrm{O} / \mathrm{FA}(20 \mathrm{~mL} ; 0.1 \% / 2 \%$; v/v/v; $\mathrm{SAX} 3$ ) flush dried between eluents. Fractions were dried under nitrogen (DEE, THF: $30^{\circ} \mathrm{C}$, toluene: $70^{\circ} \mathrm{C}$ ). Separation on silica was conducted using a sub-sample $(\sim 0.1 \mathrm{~g})$ of fraction SAX 0 which was diluted in $n$-hexane $(100 \mu \mathrm{L})$ and applied to a sequentially pre-conditioned $(10 \mathrm{~mL}$, THF; $10 \mathrm{~mL}, \mathrm{DCM} ; 10 \mathrm{~mL}$, $n$-hexane) silica SPE cartridge (Isolute ${ }^{\circledR} \mathrm{SI}, 1 \mathrm{~g}, 6 \mathrm{~mL}$, Biotage $\mathrm{AB}$, Sweden). Fractions were sequentially eluted from the silica cartridge under vacuum (-0.1 bar) with the following solvents: $n$-hexane $(10 \mathrm{~mL}$; S0), DCM/n-hexane $(20 \% / 80 \%, v / v ; 10 \mathrm{~mL}$; $\mathrm{S} 1), \mathrm{DCM} / \mathrm{h}$ exane $(50 \% / 50 \%, v / \mathrm{v} ; 10 \mathrm{~mL}$; S2), DCM $(10 \mathrm{~mL}$; $\mathrm{S} 3$ ), and THF (10 mL; S4). Solvent was subsequently removed under nitrogen at a suitable temperature $\left(30^{\circ} \mathrm{C}\right)$.

Method Development- Development of separation procedures was focused first on achieving maximum recovery from the separation of a crude oil. Alaska North Slope (ANS) crude oil was subject to the framework: $\mathrm{SCX} \rightarrow \mathrm{SAX} \rightarrow$ Silica, and a solvent scheme developed to enable maximum recovery. Experimentation utilising IEX highlighted substantial variations in isolated fractions (gravimetric and compositional) depending on the order in which SCX and SAX separations were conducted (Figure $\mathrm{S} 1$ ); recovery of basic compounds was considerably lower if the sample had been previously subject to SAX chromatography. 
Please note: This is a pre-publication copy of the final article published in Analytical Chemistry. Readers are advised to consult the final published article before citing the work.

On introduction of crude oil samples to SCX cartridges, flocculation and subsequent deposition of a black ether-insoluble material (Figure S2A) was observed. Utilisation of a toluene wash

readily solubilised and eluted (Figure S2C) the bulk of the deposited material and resulted in an "asphaltene" type fraction.

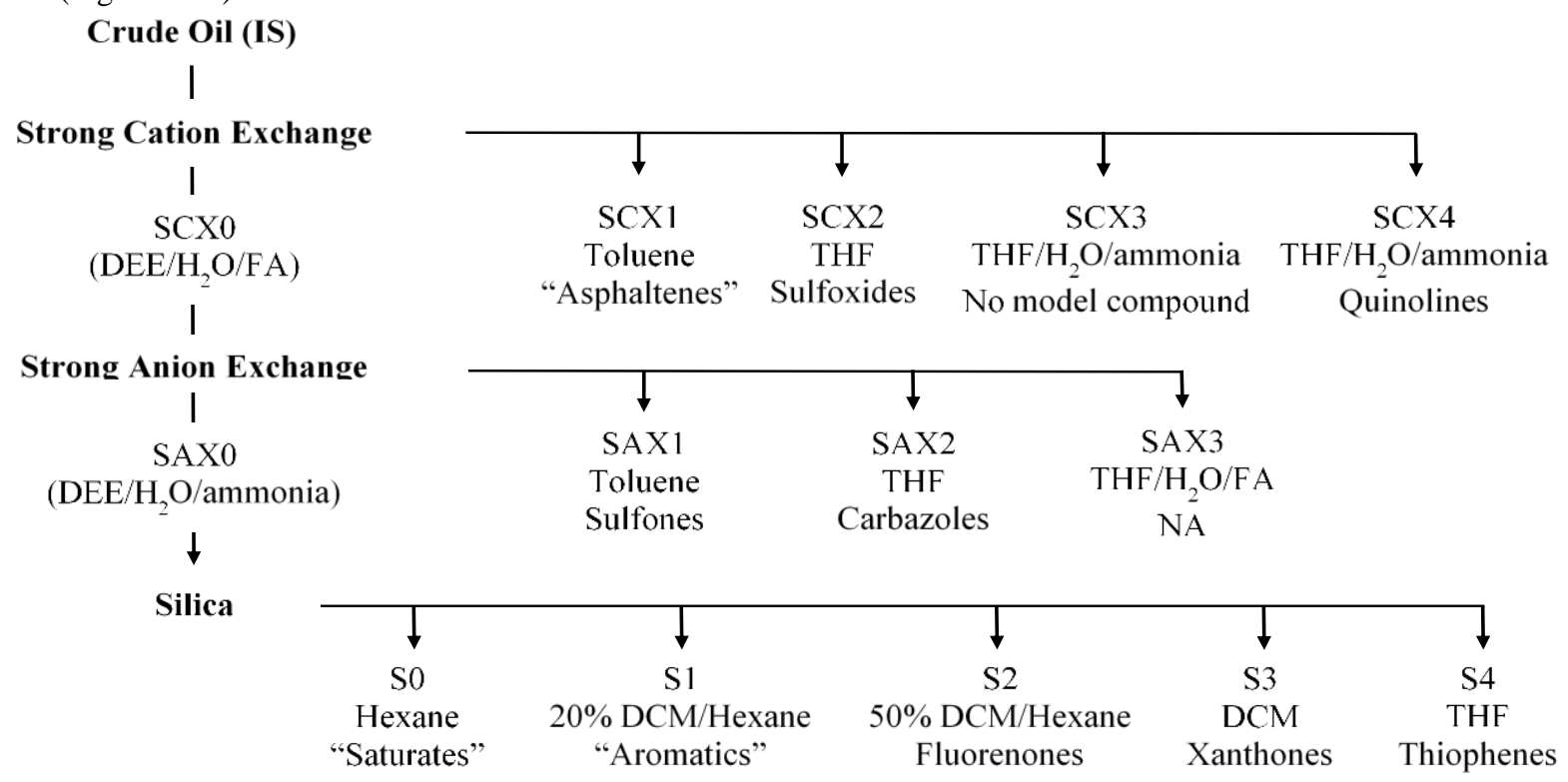

Figure 1. Flow diagram for the compound class separation of crude oils into discrete compound classes. Fraction numbers are related to solid phase extraction phase, e.g. SCX = strong cation exchange, $\mathrm{SAX}=$ strong anion exchange, $\mathrm{S}=$ silica; suffix 0 refers to non-retained fractions and positive integers to the order of elution from SPE cartridges. Elution solvents and location of model compound elutions are listed along with the fraction label

(Figure S2B). Experimentation with previously employed solvents for the removal of basic material, modified methanol ${ }^{[43,}$ ${ }^{44]}$ and binary mixtures of THF/DCM ${ }^{[52]}$, showed these to be a poor choice; solubilising only a small fraction of basic materials. Neat THF proved the most appropriate, capable of solubilising the higher volumes of modifier necessary to elute the more strongly exchanged compounds. Subsequent introduction of the un-retained SCX fraction to an SAX cartridge facilitated isolation of petroleum acids. Further fractionation of the materials not retained by SCX and SAX was required to separate "neutral polar" compounds from bulk hydrocarbon material. Four phases commonly used in crude oil separations: unbonded silica $(\mathrm{SiOH})$, alumina $\left(\mathrm{Al}_{2} \mathrm{O}_{3}\right)$, and both cyano $\left(\left(\mathrm{CH}_{2}\right)_{3} \mathrm{CN}\right)$, and amino propyl $\left(\left(\mathrm{CH}_{2}\right)_{3} \mathrm{NH}_{2}\right)$ functionalised silica, were evaluated for their ability to separate such un-retained fractions. Maximum retention of aromatic and NSO containing compounds was observed with separation on silica. An $n$-hexane-DCM stepped gradient was employed to separate non-ionic polar components from the hydrocarbons. Elutions of $n$-hexane and $20 \%$ DCM- $n$-hexane allowed isolation of saturate and aromatic hydrocarbon fractions with subsequent elutions removing the more polar constituents.

"Model" Compound Mixture- Method validation was achieved by the application of the separation scheme to a mixture of 19 model compounds (Table S1). Model compounds represented polar and apolar compound classes known to be present in crude oils and included: PAHs, sulphides, sulfoxides, thiophenes, sulfones, furans, phenols, ketones, carbazoles, quinolines, nitro compounds, and NA (including additionally functionalised acids). This mixture was separated both as the neat mixture and as a mixture spiked into ANS crude oil. Briefly, $1 \mathrm{~mL}$ of the model compound mixture $\left(0.2 \mathrm{mg} \mathrm{mL}^{-1}\right.$ per component) was syringed into each of two glass vials. The solvent was evaporated $\left(\mathrm{N}_{2}, 30{ }^{\circ} \mathrm{C}\right)$ and $\sim 0.5 \mathrm{~g}$ of Alaska North
Slope (ANS) crude oil was added to one of the vials and sonicated (10 mins, $30^{\circ} \mathrm{C}$, Camlab, CamSonix C275T). Both vials were then subject to the separation as described previously. Collected fractions were analysed by gas chromatography-mass spectrometry (GC-MS) and the compounds located within the scheme, allowing the prediction of compound class location. Quantitation of compounds within fractions was made by comparison with a 5-point calibration series for each compound in the mixture $\left(1 \mathrm{mg} \mathrm{L}^{-1}, 5 \mathrm{mg} \mathrm{L}^{-1}, 10 \mathrm{mg} \mathrm{L}^{-1}, 15 \mathrm{mg} \mathrm{L}^{-1}, 25 \mathrm{mg} \mathrm{L}^{-}\right.$ ${ }^{1}$ ) and data were processed using the Mass Hunter suite (version B.07.00; Agilent Technologies, UK). Acids were analysed as their trimethylsilyl esters (excess of BSTFA added to injection vials prior to analysis and left to stand for 1 hour). Having optimised the separation scheme for recovery from the ANS crude oil and validated it using a suite of model compounds, both alone and when dosed into the ANS crude oil (triplicate separations), the method was applied to five crude oils of varying API gravity $\left(12.1-38.3^{\circ}\right)$.

Instrumental Analysis- GC-MS analyses were conducted using a 7890A gas chromatograph interfaced with a 7683B autosampler and a 5975A quadrupole mass selective detector (MSD; (Agilent Technologies, UK). Helium carrier gas was used (constant flow; $1.0 \mathrm{~mL} \mathrm{~min}^{-1}$ ) and samples $(1 \mu \mathrm{L})$ were injected into a split/splitless injector (splitless; $250{ }^{\circ} \mathrm{C}$ ) onto an Rxi-1ms (Crossbond $100 \%$ dimethyl polysiloxane) fused silica column $(30 \mathrm{~m} \times 0.25 \mathrm{~mm} \times 0.25 \mu \mathrm{m}$; Restek Corporation, USA). The oven was programmed from $40^{\circ} \mathrm{C}$ ( 1 min hold) to $300{ }^{\circ} \mathrm{C}$ at $10{ }^{\circ} \mathrm{C} \min ^{-1}$ (10 min hold). The MS transfer line was maintained at $280{ }^{\circ} \mathrm{C}$ and the ion source at $250^{\circ} \mathrm{C}$. Mass spectra were scanned in electron impact (EI) full scan mode $(-70 \mathrm{eV})$ from $\mathrm{m} / \mathrm{z}, 50-550$ and recorded using Chemstation (version F.01.01.2317; Agilent Technologies, UK). System suitability was routinely checked using the internal calibration procedure and PFTBA calibration compound. 
Please note: This is a pre-publication copy of the final article published in Analytical Chemistry. Readers are advised to consult the final published article before citing the work.

Comprehensive two dimensional gas chromatography-mass spectrometry (GC×GC-MS) analyses were performed using a 7890A Gas Chromatograph (Agilent Technologies, UK) interfaced with a Zoex ZX2 GCXGC cryogenic modulator (Zoex, Houston, TX, USA) and a Markes/Almsco Bench Tof $\mathrm{dx}^{\mathrm{TM}}$ time of flight mass spectrometer (Markes International, Llantrisant, Wales, UK). Helium carrier gas was used (constant

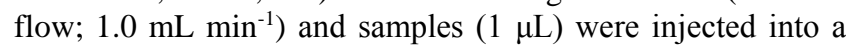
split/splitless injector (splitless; $250{ }^{\circ} \mathrm{C}$ ). The first dimension column was a $100 \%$ dimethyl polysiloxane $(60 \mathrm{~m} \times 0.25 \mathrm{~mm} \times$ $0.25 \mu \mathrm{m}) \mathrm{Rxi}{ }^{\circledR}-1 \mathrm{~ms}$ (Restek, Bellefonte, USA), and the seconddimension column was a $50 \%$ phenyl polysilphenylene siloxane $(2.5 \mathrm{~m} \times 0.25 \mathrm{~mm} \times 0.25 \mu \mathrm{m})$ BPX50 (SGE, Melbourne, Australia). The oven was programmed from $35^{\circ} \mathrm{C}$ ( 1 min hold $)$, to $120{ }^{\circ} \mathrm{C}$ at $5^{\circ} \mathrm{C} \mathrm{min}{ }^{-1}$, then to $280{ }^{\circ} \mathrm{C}$ at $2^{\circ} \mathrm{C} \mathrm{min}{ }^{-1}$, and finally to $320^{\circ} \mathrm{C}$ at $5^{\circ} \mathrm{C} \min ^{-1}$ (10 min hold). The hot jet was programmed to a temperature of $105^{\circ} \mathrm{C}(1 \mathrm{~min}$ hold $)$, heated to $190^{\circ} \mathrm{C}$ at 5 ${ }^{\circ} \mathrm{C} \min ^{-1}$, then to $350{ }^{\circ} \mathrm{C}$ at $2{ }^{\circ} \mathrm{C} \mathrm{min}^{-1}$, and finally to $390{ }^{\circ} \mathrm{C}$ at 5 ${ }^{\circ} \mathrm{C} \min ^{-1}$. The secondary oven was heated from $85^{\circ} \mathrm{C}(1 \mathrm{~min}$ hold) to $170{ }^{\circ} \mathrm{C}$ at $5{ }^{\circ} \mathrm{C} \mathrm{min}^{-1}$, and finally to $330{ }^{\circ} \mathrm{C}$ at $2{ }^{\circ} \mathrm{C} \mathrm{min}$ ${ }^{1}$ (10 min hold). The modulation times were 4 and $5 \mathrm{~s}$ (sample dependent). The MS transfer line temperature was maintained at $300{ }^{\circ} \mathrm{C}$ and the ion source at $250{ }^{\circ} \mathrm{C}$. Mass spectrometric parameters were as follows: ionisation energy $-70 \mathrm{eV}$, scan speed $50 \mathrm{~Hz}$, recorded mass range $50-550 \mathrm{~m} / \mathrm{z}$. Data was collected in ProtoTof (Markes International, Llantrisant, Wales, U.K.) and processed with GC Image (version 2.3; Zoex, Houston, TX, USA).

Ultra high performance liquid chromatography high-resolution accurate mass-mass spectrometry (uHPLC/HRAM-MS) was carried out using a U3000 uHPLC liquid chromatography system interfaced with a Q Exactive Focus mass spectrometer fitted with a heated electrospray ionisation source (HESI II) with Xcalibur 3.0.63 software used for data acquisition and all processing (Thermo Scientific, UK). Chromatographic separation was carried out using a ACE Excel 3 SuperC18 (100 x $2.1 \mathrm{~mm})$ column (Advanced Chromatography Technologies Ltd, UK) at $70^{\circ} \mathrm{C}$ with an eluent flow rate of $500 \mu \mathrm{L} \mathrm{min}^{-1}$. Injection volume was $10 \mu \mathrm{L}$ with a needle wash of methanol $(\mathrm{MeOH})$. The solvent gradient for the analysis of basic fractions was as follows:
$0.1 \% \mathrm{FA}(\mathrm{aq}) / 0.1 \% \mathrm{FA}$ in $\mathrm{MeOH} / 0.1 \% \mathrm{FA}$ in acetonitrile/ IPA (90:5:5:0 to 0:5:5:90; 30-minute runtime). All solvents were LCMS grade (Sigma-Aldrich Company Ltd., U.K). The mass spectrometer settings were: nitrogen sheath, auxiliary, and sweep gas $(53,14$, and 3 Arbitrary units, respectively), vaporiser temperature $\left(300{ }^{\circ} \mathrm{C}\right)$, polarity (positive or negative ion), spray voltage $(+3500 /-2500 \mathrm{~V})$, capillary temperature $\left(270^{\circ} \mathrm{C}\right)$ and S-lens RF level (50). The resolution was set at 70,000 at $m / z, 200$ with a full scan $(m / z, 100-1000)$, AGC target $\left(1 \mathrm{e}^{6}\right.$, automatic gain control) and micro scans (1). External mass calibration $(\leq 3 \mathrm{ppm})$ was used with auto-calibration using Pierce LTQ Velos ESI positive ion calibration solution (n-butylamine, caffeine, MRFA, and Ultramark 1621 (Thermo Fisher Scientific, UK)) and Pierce LTQ Velos ESI negative ion calibration solution (sodium dodecylsulfate, sodium taurocholate and U1tramark 1621 (Thermo Fisher Scientific, U.K.).

\section{RESULTS AND DISCUSSION}

Separation of Model Compounds- Separation and quantitation of nineteen model compounds representing twelve compound classes known to be present in crude oils, enabled us to identify where compound classes eluted within the scheme (Figure 1). Separations were performed in triplicate to assess method repeatability. Overall, the method performed well, with all but three compounds (representing 3 classes) being isolated entirely within individual fractions. (It was important to perform the separation exactly as described (Experimental); if variations to the method are necessary due to supply of phases etc., the 'model' compound mixture can be used to re-check the elution of the fraction types).

Initial separation using SCX SPE resulted in the successful isolation of two of the model compounds. The SCX phase, a polymerically bonded benzene sulfonic acid functional group, provided a mixed mode separation with both ionic and hydrophobic interactions facilitating retention. The use of such phases for the isolation of benzoquinoline and other pyridine derivatives has been reported previously with similar success ${ }^{[44,52,53]}$. As with previous studies, benzoquinoline was isolated completely in the basic fractions (Figure 2C; SCX4).

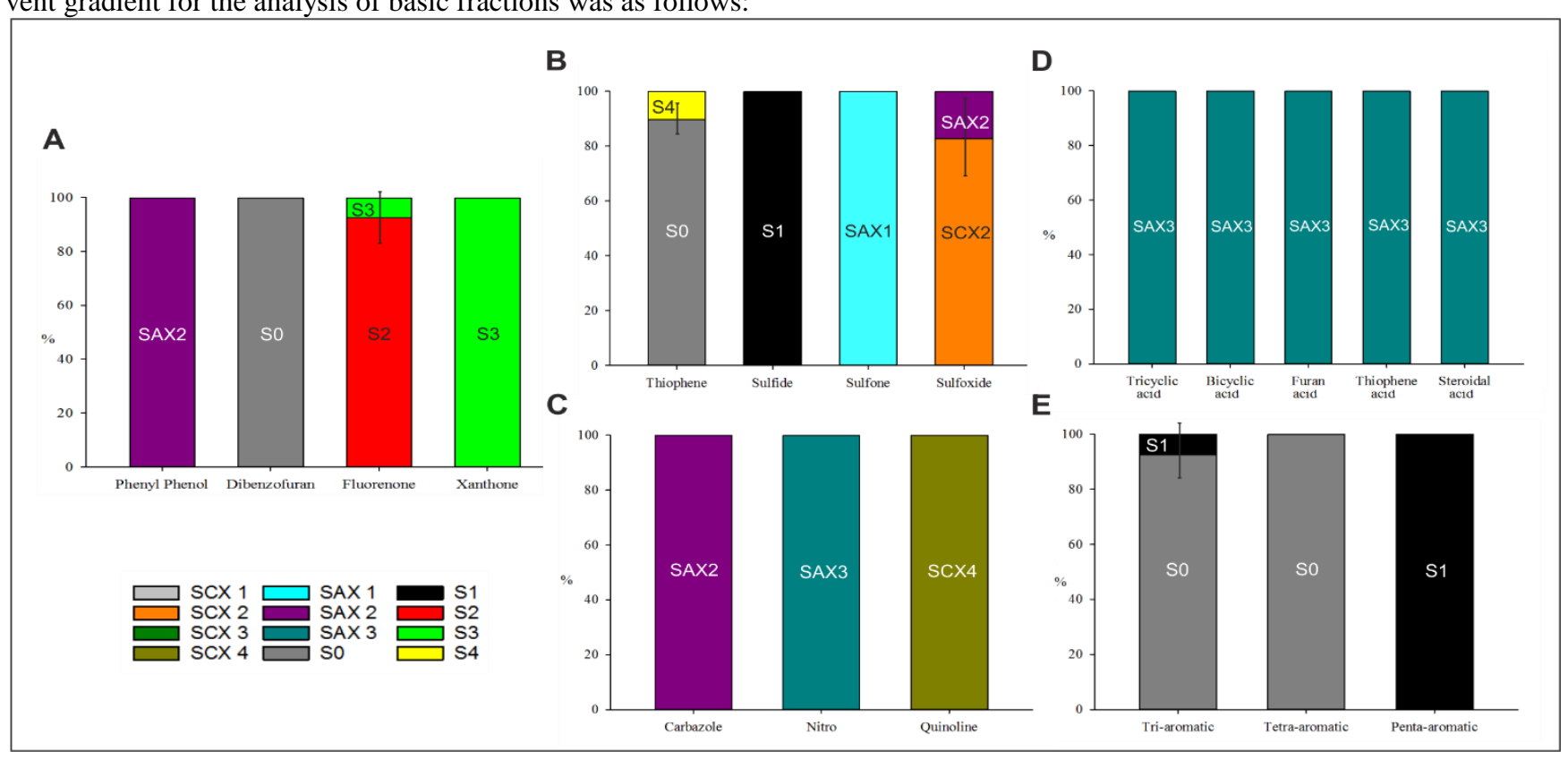


Please note: This is a pre-publication copy of the final article published in Analytical Chemistry. Readers are advised to consult the final published article before citing the work.

Figure 2. Averaged gravimetric data ( $n=3$; error bars represent separation of compounds between fractions) illustrating which chromatographic fraction contained each of the model compounds. Compounds are grouped into charts of similar compound class: Oxygen-containing (non-acid) compounds (A), sulphur-containing compounds (B), nitrogen-containing compounds (C), acids (D), and aromatic compounds (E). Single coloured bars indicate a model compound was recovered in a single fraction.

Analysis of fractions by GC-MS showed no division of the quinoline model compound between the two basic fractions, indicating strong ionic interactions between the basic nitrogen compound and the chromatographic phase.

Toluene was used to elute fraction SCX1 for practical reasons as it solubilised material suspected to be 'asphaltenic' in nature that was observed to deposit upon introduction of some crude oil samples to the SCX SPE cartridge. Inclusion of asphaltene model compounds ${ }^{[54,55]}$ in the test mix may give an indication as to whether this fraction is asphaltenic or whether the model compounds behave in the same manner as crude oil components when separated in this way. The sulfoxide model compound was recovered using THF from both of the IEX cartridges (SCX2 and SAX2; Figure 2B).

Chromatography utilising the SAX SPE cartridge resulted in the successful isolation of all the carboxylic acids in a single fraction (SAX3; Figure 2D). Ionic interactions between carboxylic acids and the quaternary amine phase appear to be unaffected by the presence of other functional groups or structural features. Retention of carbazoles and phenols to anion exchangers has been reported previously ${ }^{[43]}$ but, was considered counterproductive. In this study however, exploitation of these interactions allowed isolation of carbazoles (SAX2; Figure 2C) and phenols (SAX2; Figure 2A) within one fraction with no carryover into later fractions observed in any instance. The nitroindole compound was also recovered in the SAX3 fraction suggesting the addition of the nitro group increases the dissociation of the indole functionality, thus increasing the ionic interactions with the phase.

Normal phase chromatography conducted on silica provided separation of those neutral compounds which were not retained by the SCX or SAX SPE cartridges. Quantitation of dibenzothiophene in the silica fractions revealed the compound to be divided between fractions S0 and S4 (Figure 2B). Despite subsequent elution with increasingly polar mixtures of $n$-hexane and DCM, $10 \%$ of the compound was only collected upon elution with THF. Whist this suggested the method did not provide fractions ideally suitable for quantitation of sulphur compounds, subsequent separations of crude oils resulted in an S4 fraction composed of a number of thiophene and other sulphur containing compounds. Separation of oxygen compounds on silica was successful with furan, fluorenone and xanthone eluting in separate fractions ( $\mathrm{S} 0, \mathrm{~S} 2$ and S4 respectively; Figure 2A). Dibenzofuran eluted in fraction S0, indicating minimal retention of the compound to the silica phase. Similarly, aromatic hydrocarbon model compounds eluted in fractions S0 and S1, indicating limited retention (Figure 2E). Increasing numbers of rings resulted in an increase in retention, with benzo $(a)$ pyrene eluting within fraction $\mathrm{S} 1$.

Authentic model compounds were then spiked into the ANS crude oil in order to investigate the effect of the matrix on compound separation. Only minor differences were observed in the distribution of model compounds (Figure S3) when the chromatographic scheme was applied to the model compounds in a crude oil matrix compared to the model compounds mixture alone. In particular, no matrix effects were found for sulfide, sulfoxide, carbazole, phenol, and acidic and basic fractions. Dibenzothiophene sulfone eluted later in the scheme, divided slightly between fractions SAX1 and SAX 2 when dosed into the crude oil. Aromatic compounds eluted later, with penta- and tetra- aromatic compounds eluting completely in fractions S1. Separation of phenanthrene remained largely unchanged, but tetra- and penta- aromatic compounds eluted later (S1 rather than S0). Separation of oxygen containing compounds also showed a slight increase in retention with dibenzofuran eluting in both fractions S0 (83\%) and S1 (17\%). Fluorenone and xanthone eluted later with the former separating between fractions S2 (88\%) and S3 (12\%) and the latter between fractions S3 (9\%) and S4 (91\%). Finally, thiophene splitting appeared reduced with $>98 \%$ present within fractions S0 and only slight retention and elution in fraction S4.

Application to Crude Oil Samples- Separation procedures were further evaluated by the fractionation of five crude oils (Table S2). Total recoveries from the separation of the five oils (Table 1) were good (77.4-97.5\%) and very good (92.6-102.5 $\%)$ once corrected for simple evaporative losses $\left(\mathrm{N}_{2}\right.$ blowdown; Figure S4). This demonstrates the ability of the method to allow isolation of high molecular weight/highly polar material, thus allowing almost complete sample recovery from subsequent separations. Previously reported large scale fractionations tend to suffer from low recoveries ${ }^{[42,43]}$. Excellent recovery of the TJP crude (97.5\%; API gravity $12.1^{\circ}$ ), with a low volatiles content supports this further, also indicating that losses due to irreversible adsorption were low compared to other methods. Recoveries obtained by Willsch et al. ${ }^{[42]}$ varied from $56.6 \%$ $76.3 \%$ with high losses likely due to irreversible adsorption on the numerous employed silica separations. Snyder et al. ${ }^{[43,56]}$ reported no recoveries, but our attempts to reproduce their separation using ANS crude oil, resulted in a recovery of only $\sim 67 \%$. The method presented herein requires no sample pretreatment and in our hands at least, demonstrated exceptional recovery on crude oil samples with a broad range of physiochemical properties.

Table 1. Total and individual cartridge recoveries (weight \%) of crude oil samples separated in this study and recoveries corrected for losses on $\mathrm{N}_{2}$ blowdown. $\mathrm{N}_{2}$ blowdown data from sample concentration for 30 minutes at $30^{\circ} \mathrm{C}$ under a steady stream of $\mathrm{N}_{2}$.

\begin{tabular}{c|ccccc}
$\begin{array}{c}\text { Recovery } \\
(\%)\end{array}$ & $\begin{array}{c}\text { Alaska } \\
\text { North Slope }\end{array}$ & $\begin{array}{c}\text { Kuwait } \\
\text { Blend }\end{array}$ & $\begin{array}{c}\text { Brent } \\
\text { Blend }\end{array}$ & Bonga & $\begin{array}{c}\text { Tijuana } \\
\text { Pesado }\end{array}$ \\
\hline SCX & 87.0 & 91.5 & 94.1 & 86.3 & 98.6 \\
SAX & 97.4 & 95.3 & 94.1 & 96.3 & 99.7 \\
Silica & 96.4 & 95.4 & 91.4 & 92.1 & 95.6 \\
\hline Total & $\mathbf{8 2 . 7}$ & $\mathbf{8 4 . 9}$ & $\mathbf{8 3 . 1}$ & $\mathbf{7 7 . 4}$ & $\mathbf{9 7 . 5}$ \\
\hline Blowdown & 80.2 & 83.4 & 85.3 & 84.8 & 98.8 \\
\hline Corrected & 102.5 & 101.5 & 97.8 & 92.6 & 98.7
\end{tabular}

Method repeatability with regard to crude oil separations was investigated by the separation of triplicate samples of the ANS crude. Average fraction masses with standard deviations are presented in Figure S5, and show low variability with regard to individual fraction masses. A highly reproducible method for the compound class separation of crude oils that requires no specialist equipment offers advantages compared to other methods (e.g. SARA-type methods) where problems with reproducibility are common ${ }^{[30]}$.

Instrumental analysis of fractions obtained from separation of ANS crude oil- GC-MS analysis of fractions obtained from 
Please note: This is a pre-publication copy of the final article published in Analytical Chemistry. Readers are advised to consult the final published article before citing the work.

separation of ANS crude oil resulted in a series of chromatograms characterised by resolved peaks and unresolved complex mixtures (UCMs; Figure 3). Extracted ion mass chromatograms of molecular ions (e.g.: carbazole $\mathrm{m} / \mathrm{z}, 167$, dibenzothiophene $\mathrm{m} / \mathrm{z}$ 184, xanthone $\mathrm{m} / \mathrm{z} 196$, fluorenone $\mathrm{m} / \mathrm{z} 180$ ) and also $M+14 n$, revealed alkylated homologs of the parent heterocompounds. In a number of instances SPE separations resulted in fractions in which parent compounds and alkyl homologous $\left(\mathrm{C}_{1}\right.$ and $\mathrm{C}_{2}$ ) were sufficiently well resolved using conventional GCMS for identification. For example, chromatograms of xanthone (Fraction S3; Figure 3E) and dibenzothiophene (Fraction S4; Figure 3F) fractions show resolved, homologous series.

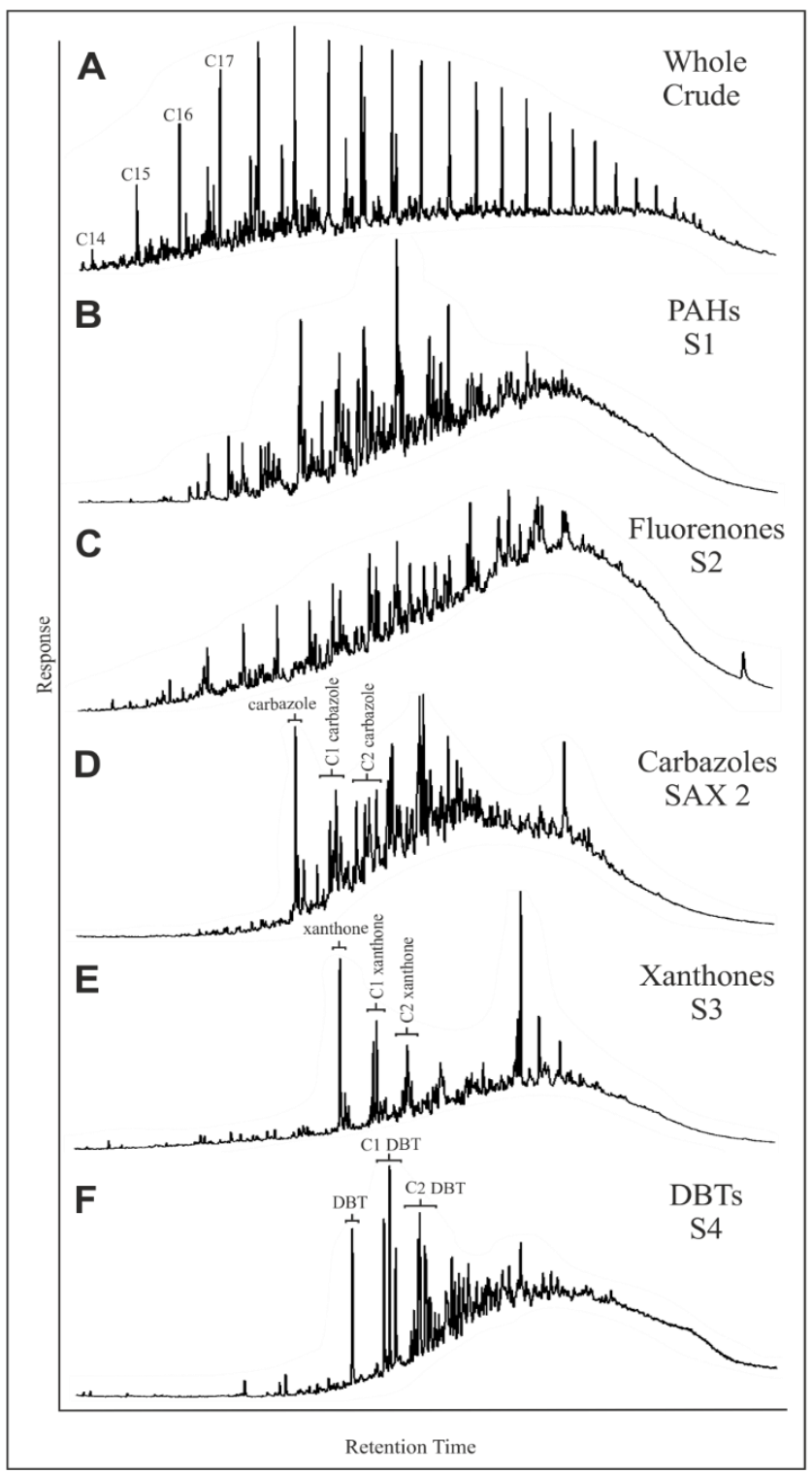

Figure 3. GC-MS TIC of ANS whole crude oil (A) and fractions obtained SPE: Fraction S1 (PAHs; B), Fraction S2 (fluorenones; C), Fraction SAX 2 (carbazoles; D), Fraction S3 (xanthones; E), and Fraction S4 (dibenzothiophenes; F).

However, to obtain better quality mass spectra for later eluting compounds within the GC-MS UCM, GC $\times$ GC-MS was used. The GC $\times$ GC-MS Total Ion Chromatogram (TIC) from the analysis of the carbazole fraction (Fraction SAX2; Figure 4A) obtained from ANS crude oil shows a homologues series of wellresolved peaks (Figure 4B). Closer inspection of the TIC shows
Fraction SAX2 represents an almost exclusive separation towards carbazole and its alkyl homologues. Identity of the parent carbazole was confirmed by co-injection of the authentic standard and comparison of the two dimensional retention position, and mass spectrum (Figure S6 A and Ai). Assignments of $\mathrm{C}_{1}$, and $\mathrm{C}_{2}$ carbazoles were made by comparison with library (NIST) spectra (Figure S6 B and Bi and S6 C and Ci) and further identifications made based on chromatographic co-ordinates of peaks and mass spectral interpretation (Figure S6 D-F). GC $\times$ GC-MS analysis of the thiophene Fraction (S4) obtained from the separation of ANS crude oil, resulted in excellent chromatographic separation (Figure 5A). The TIC (Figure S7A) indicates that the DBT series are the most abundant components

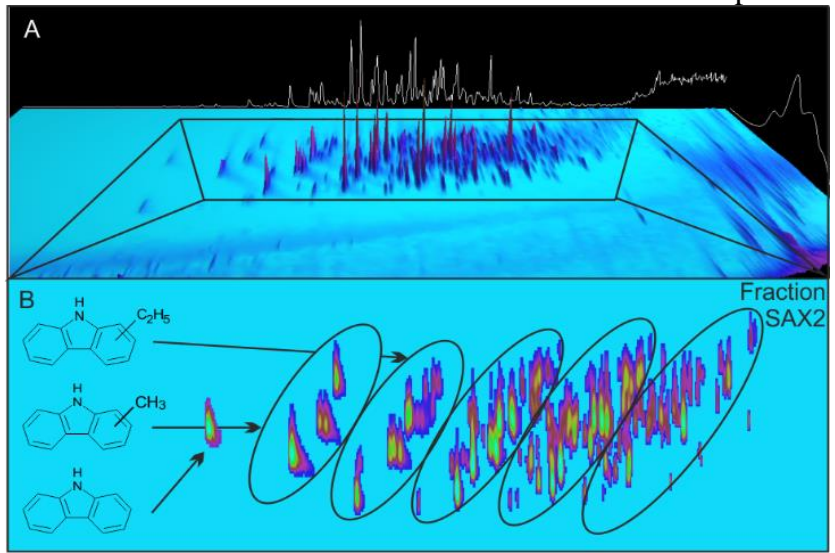

Figure 4. GC $x$ GC- MS TIC of Fraction SAX2 from the separation of ANS crude oil with carbazole series highlighted (A), and EIC of carbazole and $\mathrm{C}_{1-5}$ homologs $(\mathrm{m} / \mathrm{z}, 167,181,195,209,223,237)$ (B).

within the mixture and. Alkylated homologues are likely responsible for the observed UCM in the GC-MS TIC (Figure $3 F)$. Extracted ion monitoring allowed clear, distinguishable mass spectra to be obtained for individual $\mathrm{C}_{1-4}$ DBTs (Figure S8). Substantial complexity accompanying the later eluting $\mathrm{C}_{5}$ dibenzothiophenes results in significant co-elution even when GC $\times$ GC-MS was used. Identification of the parent DBT was confirmed by co-injection of the authentic standard, allowing comparison of both mass spectra (Figure S8 A and Ai), and primary and secondary GC retention indices. Assignment of individual methyl, and dimethyl or ethyl DBT isomers was not possible without co-injection of authentic compounds. However, comparison of mass spectra with those of the NIST database (Version 2.0; Figure S8B and $\mathrm{Bi}$ and $\mathrm{S} 6 \mathrm{C}$ and $\mathrm{Ci}$ ) provided sufficient confirmation of the presence of the series. 
Please note: This is a pre-publication copy of the final article published in Analytical Chemistry. Readers are advised to consult the final published article before citing the work.

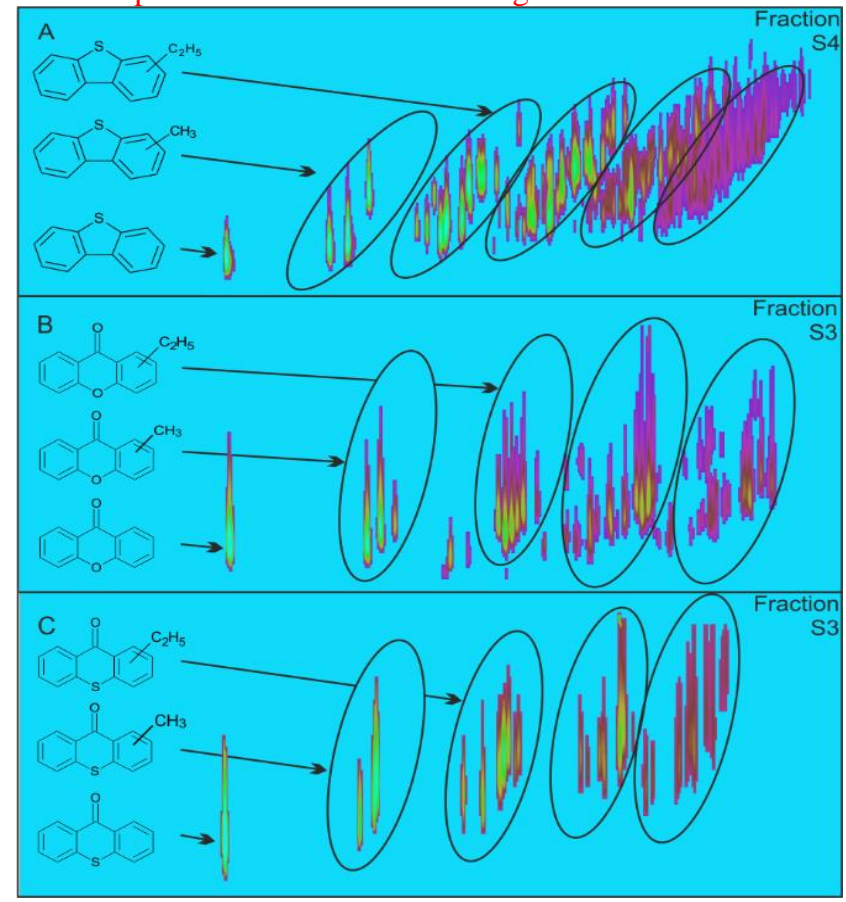

Figure 5. GC $\times$ GC-MS EICs for DBT and $\mathrm{C}_{1-5}$ DBT homologs $(\mathrm{m} / \mathrm{z}$ $184,198,212,226,240,254$, respectively) from the analysis of the thiophene Fraction (S4; A) and for xanthone and thioxanthone, and respective $\mathrm{C}_{1-4}$ homologs $(\mathrm{m} / \mathrm{z}, 196,210,224,238,252$ and $\mathrm{m} / \mathrm{z}, 212$, $226,240,254,268$, respectively) from the analysis of the xanthone Fraction (S3; B and C respectively) from the separation of the ANS crude oil

Xanthone and alkyl homologs were found to be present in Fraction S3 of the ANS crude oil as determined by GC-MS (Figure 3E) and GC $\times$ GC-MS (EIC Figure 5B; TIC Figure S7B). Coinjection of an authentic xanthone standard allowed confirmation of parent molecule (Figure S9 A and Ai) and fragmentation pathway (Figure S10) also supported compound identification. Library spectra for alkylated xanthones were not available in the NIST library (Version 2.0). Studies concerning the presence of xanthones in crude oils are uncommon, with perhaps only two reports in the literature $\left({ }^{[9,57]}\right)$. Without available standards or model spectra to confirm identifications, assignments of $\mathrm{C}_{1-5}$ alkyl xanthones were made on two dimensional retention time, molecular mass and interpretation of spectra. Mass spectral studies of the alkyl xanthones and indeed homologues of other oxygenated PAHs (e.g., benzofurans, fluorenones) are rare ([58${ }^{60]}$ ) and none have interpreted the mass spectra of alkylated structures. Interestingly, mass spectra of the methyl substituted compounds did not exhibit the common $\mathrm{M}^{+\cdot}-15$ losses (Figure S9 B), indicative of the loss of a methyl substituent $\left(\mathrm{CH}_{3}\right)$. Instead, these appeared to lose a neutral $\mathrm{CHO}\left(\mathrm{M}^{+\cdot}-29\right)$ fragment and to undergo subsequent rearrangement with the alkyl substituent being incorporated into the benzene ring to which it was attached, forming a 7 membered ring (postulated fragmentation illustrated in Figure S11).

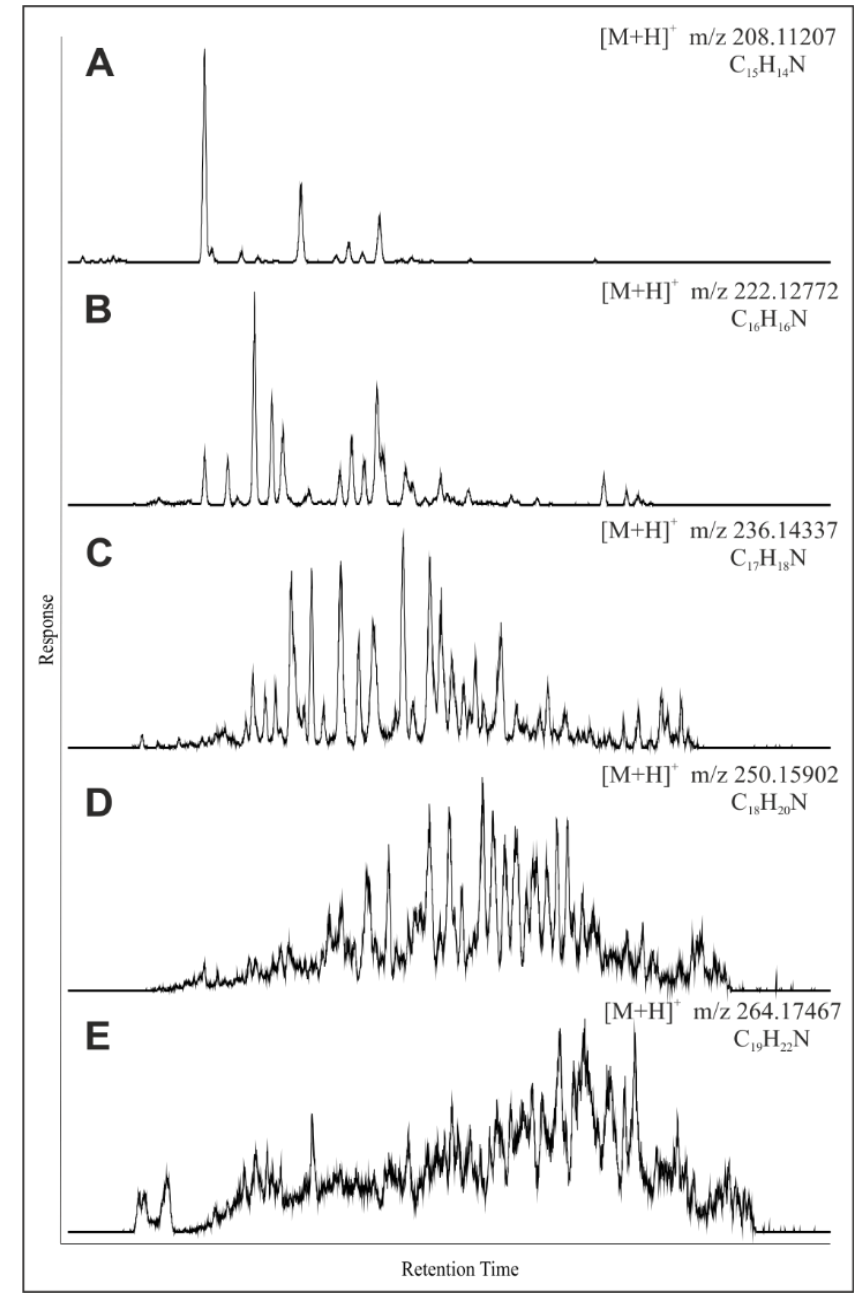

Figure 6. LC/HRAM-MS EIC's from the analysis of Fraction SCX4 from the separation of the ANS crude oil. Presented EIC's represent one of the more abundant, lower molecular weight series. Accurate masses (protonated) are shown, alongside assigned molecular formulae.

However, structures possessing a larger degree of alkylation $\left(\mathrm{C}_{2}-\mathrm{C}_{5}\right)$ do appear to lose a methyl group during fragmentation which is then followed by a loss of CHO (Figure S8 C-E) and subsequent characteristic losses.

Thioxanthones and alkyl thioxanthones not previously reported in crude oils, were tentatively identified in Fraction S3 of the ANS crude oil (Figure 5C). Identification of the thioxanthone parent molecule was facilitated by mass spectral comparison to a NIST library spectrum (Reverse Match 903; Probability 97\%; Figure S12 A-Ai). Inspection of mass spectra of thioxanthone and alkyl thioxanthones (Figure S12 B-E) reveal similar fragmentation patterns to that of the xanthone series and the fragmentation is rationalised in Figure S13.

Not all fractions were amenable to analysis by GC-based techniques. Application of LC/HRAM-MS to the benzoquinoline fraction (SCX4) revealed a highly complex mixture. Mass spectral ions primarily presented with even masses (indicative of an odd number of $\mathrm{N}$ ), high ring double bond equivalency and occurred within the mass range $m / z, 150-1400$. EICs of a more abundant series (Figure 6) demonstrated separation of structural isomers, allowing MS-MS experiments to be conducted on individual compounds. Generation of molecular formulae from 
Please note: This is a pre-publication copy of the final article published in Analytical Chemistry. Readers are advised to consult the final published article before citing the work.

accurate mass measurements suggested the series to be $\mathrm{N}$-containing aromatics with increasing degrees of alkylation. These identifications were further supported by the elution of the benzoquinoline model compound within this fraction, indicating the likely presence of N-containing aromatics. However, it can be seen that even at relatively low molecular masses (e.g. $\mathrm{m} / \mathrm{z}$ 250; Figure 6D), the number of isomers still produces a challenge to LC resolution using a one dimensional separation method such as LC/HRAM-MS

\section{CONCLUSION}

Previously reported methods for the molecular class separation of crude oil have been shown to suffer from many issues. Here we have demonstrated a robust method for the chemical separation of crude oils. Analysis of the resultant fractions by appropriate gas and liquid chromatographic techniques demonstrated that the method is capable of providing well defined fractions of various compound classes, allowing rapid identification of common and rarely reported crude oil NSO compounds (e.g. carbazoles, DBTs, xanthones, thioxanthones, NA, hydrocarbons). uHPLC-HRAM-MS analysis of ionic fractions was shown to be a promising technique for the separation and characterisation of such fractions, allowing assignment of elemental formula through accurate mass measurements and an insight into the structural complexity of individual molecular formulae.

\section{ASSOCIATED CONTENT Supporting Information}

Mass spectra of all identified compounds are provided in the supporting information (Figures S6-S11). This information is available free of charge via the Internet http://pubs.acs.org.

\section{AUTHOR INFORMATION Corresponding Author}

*Phone: +44 (0)1752 584557. Fax: +44 (0)1752 584710. E-mail: srowland@plym.ac.uk

\section{ACKNOWLEDGMENT}

Funding for this research was provided by Kernow Analytical Technology Ltd. (Cornwall, UK) and the University of Plymouth to whom we are extremely grateful. We would also like to thank Dr C. Anthony Lewis for invaluable discussions and Dr Michael Wilde for assistance with GCxGC operation. Finally, we would like to thank BP, Shell and the Nigerian National Petroleum Corporation for crude oil samples.

\section{REFERENCES}

(1) von Muhlen, C., E.C. de Oliveira, C.A. Zini, E.B. Caramao, and P.J. Marriott. Energy \& Fuels. 2010, 24, 3572-3580.

(2) von Muhlen, C., C.A. Zini, E.B. Caramao, and P.J. Marriott. J Chromatogr A. 2006, 1105, 39-50.

(3) Mills, G.A., E.R. Boedeker, and A.G. Oblad. J Am Chem Soc. 1950, 72, 1554-1560.

(4) Bauserman, J.W., G.W. Mushrush, and D.R. Hardy. Ind Eng Chem Res. 2008, 47, 2867-2875.

(5) Dutson, S.M., G.M. Booth, G.B. Schaalje, R.N. Castle, and R.E. Seegmiller. Environmental Toxicology and Chemistry. 1997, 16, 2113-2117.

(6) Kropp, K.G. and P.M. Fedorak. Can J Microbiol. 1998, 44, 605622.

(7) Li, M., T.G. Wang, S. Shi, K. Liu, and G.S. Ellis. Marine and Petroleum Geology. 2014, 57, 403-417.
(8) Pan, Y.H., Y.H. Liao, Q. Shi, and C.S. Hsu. Energy \& Fuels. 2013, 27, 2960-2973.

(9) Oldenburg, T.B.P., H. Wilkes, B. Horsfield, A.C.T. van Duin, D. Stoddart, and A. Wilhelms. Org Geochem. 2002, 33, 595-609.

(10) Bennett, B. and S.R. Larter. Org Geochem. 2000, 31, 117-125.

(11) Petkova, N., M. Angelova, and P. Petkov. Oxid. Commun. 2009, 32, 643-653.

(12) Jin, P., S. Nesic, and H.A. Wolf. Surface and Interface Analysis. 2015, 47, 454-465.

(13) Scarlett, A., C.E. West, D. Jones, T. Galloway, and S.J. Rowland. Science of the Total Environment. 2012, 425,

(14) Scarlett, A.G., H.C. Reinardy, T.B. Henry, C.E. West, R.A. Frank, L.M. Hewitt, and S.J. Rowland. Chemosphere. 2013, 93, 415-20.

(15) Sutton, P.A. and S.J. Rowland. Energy \& Fuels. 2014, 28, 5657-5669.

(16) Jewell, D.M., E.W. Albaugh, B.E. Davis, and R.G. Ruberto. Industrial \& Engineering Chemistry Fundamentals. 1974, 13, 278 282.

(17) Suatoni, J.C. and R.E. Swab. J Chromatogr Sci. 1975, 13, 361366.

(18) Galya, L.G. and J.C. Suatoni. Journal of Liquid Chromatography. 1980, 3, 229-242.

(19) Bollet, C., J.C. Escalier, C. Souteyrand, M. Caude, and R. Rosset. J Chromatogr A. 1981, 206, 289-300.

(20) Grizzle, P.L. and D.M. Sablotny. Anal Chem. 1986, 58, 23892396.

(21) Hayes, P.C. and S.D. Anderson. J Chromatogr A. 1988, 437, 365-377.

(22) Hayes, P.C. and S.D. Anderson. Anal Chem. 1986, 58, 23842388.

(23) Karlsen, D.A. and S.R. Larter. Org Geochem. 1991, 17, 603617.

(24) Robbins, W.K. J Chromatogr Sci. 1998, 36, 457-466.

(25) Fan, T. and J.S. Buckley. Energy \& Fuels. 2002, 16, 15711575 .

(26) Jiang, C.Q., S.R. Larter, K.J. Noke, and L.R. Snowdon. Org Geochem. 2008, 39, 1210-1214.

(27) Schabron, J.F., J.F. Rovani, and M.M. Sanderson. Energy \& Fuels. 2010, 24, 5984-5996.

(28) Kim, E., E. Cho, S. Moon, J.-I. Park, and S. Kim. Energy \& Fuels. 2016, 30, 2932-2940.

(29) Bissada, K.K., J. Tan, E. Szymczyk, M. Darnell, and M. Mei. Org Geochem. 2016, 95, 21-28.

(30) Kharrat, A.M., J. Zacharia, V.J. Cherian, and A. Anyatonwu. Energy \& Fuels. 2007, 21, 3618-3621.

(31) Adams, J.J., J.F. Schabron, and R. Boysen. Energy \& Fuels. 2015, 29, 2774-2784.

(32) Boysen, R.B. and J.F. Schabron. Energy \& Fuels. 2013, 27, 4654-4661.

(33) Schabron, J.F. and J.F. Rovani Jr. Fuel. 2008, 87, 165-176.

(34) Cho, Y.J., J.G. Na, N.S. Nho, S. Kim, and S. Kim. Energy \& Fuels. 2012, 26, 2558-2565.

(35) Gaspar, A., E. Zellermann, S. Lababidi, J. Reece, and W. Schrader. Energy \& Fuels. 2012, 26, 3481-3487.

(36) Tachon, N., F. Jahouh, M. Delmas, and J.H. Banoub. Rapid Communications in Mass Spectrometry. 2011, 25, 2657-2671.

(37) Tong, J., J. Liu, X. Han, S. Wang, and X. Jiang. Fuel. 2013, 104, 365-371.

(38) Bae, E., J.-G. Na, S.H. Chung, H.S. Kim, and S. Kim. Energy \& Fuels. 2010, 24, 2563-2569.

(39) Nelson, S.D., In Biological Reactive Intermediates VI: Chemical and Biological Mechanisms in Susceptibility to and Prevention of Environmental Diseases.Ed.; P.M. Dansette, R. Snyder, M. Delaforge, G.G. Gibson, H. Greim, D.J. Jollow, T.J. 
Please note: This is a pre-publication copy of the final article published in Analytical Chemistry. Readers are advised to consult the final published article before citing the work.

Monks, and I.G. Sipess, Springer US, Boston, MA. 2001, pp 33-

43.

(40) Argyle, M. and C. Bartholomew. Catalysts. 2015, 5, 145.

(41) Sutton, P.A., B.E. Smith, and S.J. Rowland. Rapid Communications in Mass Spectrometry. 2010, 24, 3195-3204.

(42) Willsch, H., H. Clegg, B. Horsfield, M. Radke, and H. Wilkes. Anal Chem. 1997, 69, 4203-4209.

(43) Snyder, L.R. and B.E. Buell. Anal Chem. 1968, 40, 1295-1302.

(44) Conceição Oliveira, E., M.C.1. Vaz de Campos, A. Sant'Ana

Lopes, M.G. Rodrigues Vale, and E. Bastos Caramão. J Chromatogr A. 2004, 1027, 171-177.

(45) Fang, R., T.G. Wang, M. Li, Z. Xiao, B. Zhang, S. Huang, S. Shi, D. Wang, and W. Deng. Org Geochem. 2016, 91, 68-80.

(46) Oldenburg, T.B.P., H. Wilkes, B. Horsfield, A.C.T. van Duin, D. Stoddart, and A. Wilhelms. Org Geochem. 2002, 33, 595-609.

(47) Lundanes, E. and T. Greibrokk. Journal of High Resolution Chromatography. 1994, 17, 197-202.

(48) Jewell, D.M., J.H. Weber, J.W. Bunger, H. Plancher, and D.R. Latham. Anal Chem. 1972, 44, 1391-\&.

(49) McKay, J.F., P.J. Amend, P.M. Harnsberger, T.E. Cogswell, and D.R. Latham. Abstr Pap Am Chem S. 1976, 172, 53-53.

(50) Boduszynski, M., B.R. Chadha, and T. Szkutapochopien. Fuel. 1977, 56, 432-436.

(51) Jones, D.M., J.S. Watson, W. Meredith, M. Chen, and B. Bennett. Anal Chem. 2001, 73, 703-707.

(52) Simon, S., A.L. Nenningsland, E. Herschbach, and J. Sjöblom. Energy \& Fuels. 2010, 24, 1043-1050.

(53) Schmitter, J.M., I. Ignatiadis, P. Arpino, and G. Guiochon. Anal Chem. 1983, 55, 1685-1688.

(54) López-Linares, F., L. Carbognani, M.F. González, C. SosaStull, M. Figueras, and P. Pereira-Almao. Energy \& Fuels. 2006, 20, 2748-2750.

(55) Nordgård, E.L.k. and J. Sjöblom. Journal of Dispersion Science and Technology. 2008, 29, 1114-1122.

(56) Snyder, L.R., B.E. Buell, and H.E. Howard. Anal Chem. 1968, 40, 1303-1317.

(57) Bakr, M.M.Y. Journal of King Abdulaziz University; Earth sciences. 2009, 20, 127-159.

(58) Granoth, I. and H.J. Pownall. The Journal of Organic Chemistry. 1975, 40, 2088-2091.

(59) Spilker, R. and H.-F. Grützmacher. Org Mass Spectrom. 1989, 24, 47-54.

(60) Arends, P., P. Helboe, and J. Møller. Org Mass Spectrom. 1973, 7, 667-681. 